\title{
Curcumin analogue GO-Y030 inhibits STAT3 activity and cell growth in breast and pancreatic carcinomas
}

\author{
BRIAN HUTZEN ${ }^{1,2}$, LAUREN FRIEDMAN $^{1}$, MATTHEW SOBO ${ }^{1,2}$, LI LIN $^{1}$, LING CEN $^{1}$, \\ STEPHANIE DE ANGELIS ${ }^{1}$, HIROYUKI YAMAKOSHI ${ }^{3}$, HIROYUKI SHIBATA ${ }^{4}$, \\ YOSHIHARU IWABUCHI ${ }^{3}$ and JIAYUH LIN ${ }^{1,2,4,5}$
}

\begin{abstract}
${ }^{1}$ Department of Pediatrics, ${ }^{2}$ Molecular, Cellular, and Developmental Biology Program, ${ }^{3}$ Department of Organic Chemistry, Graduate School of Pharmaceutics, Tohoku University; ${ }^{4}$ Department of Clinical Oncology, Institute of Development, Aging and Cancer, Tohoku University, Sendai, Japan; ${ }^{5}$ Experimental Therapeutics Program, The Ohio State University Comprehensive Cancer Center, College of Medicine, The Ohio State University, Columbus, OH 43210, USA
\end{abstract}

Received May 6, 2009; Accepted June 24, 2009

DOI: 10.3892/ijo_00000401

\begin{abstract}
Curcumin has numerous anti-carcinogenic properties, but low bioavailability prevents its use in chemotherapeutic applications. One strategy for circumventing this problem has been the creation of synthetic analogues. We tested the efficacy of an analogue known as GO-Y030 in human breast and pancreatic cancer cells. We compared the impact of curcumin and GO-Y030 on the breast cancer cell line MDA-MB-231 and pancreatic cancer cell lines, PANC-1, HPAC and BXPC-3. Both compounds reduced cell viability and induced apoptosis, but GO-Y030 was substantially more potent. We also demonstrated that GO-Y030 was capable of interfering with STAT3, a persistently activated transcription factor in many cancer types. GO-Y030 inhibited STAT3 phosphorylation and transcriptional activity whereas comparable dosages of curcumin had little or no effect. These results indicate that GO-Y030 is a potent inhibitor of cell viability and STAT3 activation, and may thus have potential as a therapeutic agent for cancers expressing high levels of activated STAT3.
\end{abstract}

\section{Introduction}

Curcumin is a polyphenolic compound isolated from the rhizome of the perennial herb Curcuma longa. It has been part of therapeutic preparations for centuries due to its wide spectrum of beneficial activities and its safety in relatively large doses (1). Extensive research has indicated that the

Correspondence to: Dr Jiayuh Lin, Center for Childhood Cancer, The Research Institute at Nationwide Children's Hospital, Department of Pediatrics, College of Medicine, The Ohio State University, 700 Children's Drive, Columbus, OH 43205, USA

E-mail: lin.674@osu.edu

Key words: breast cancer, pancreatic cancer, curcumin, GO-Y030, STAT3 complex chemistry of curcumin allows it to influence multiple cell signaling pathways, giving it anti-inflammatory, antioxidant, chemopreventive and chemotherapeutic properties in addition to many others (2). The anti-carcinogenic properties of curcumin continue to be a subject of great interest, and evidence that it can inhibit the initiation, progression and continued survival of cancerous cells likewise continues to accumulate (2). Despite these promising findings, curcumin has yet to be approved as a chemotherapeutic agent. Testing in animal models and human clinical trials has revealed that the bioavailability of curcumin is low, owing to its poor absorption, limited tissue distribution, rapid metabolism, and subsequent elimination from the body (3). Numerous strategies have been devised to address these shortcomings, including the design and synthesis of novel structural analogues (4).

One such compound, termed GO-Y030, was synthesized by Ohori and colleagues during the course of an exhaustive screen with the dual focus of identifying the structural motifs responsible for curcumin's anti-carcinogenic properties and then designing analogues that improved upon them (5). Following their initial evaluation, GO-Y030 was found to possess potent growth-suppressive potential in several cancer cell lines, inhibiting cell growth by a factor of 8-40 times that exhibited by curcumin (5). Despite its higher efficacy, GO-Y030 also retained curcumin's relative safety. Dosages as high as $100 \mu \mathrm{M}$ were found to have minimal impact on primary human hepatocytes, and in vivo toxicity studies performed in mice revealed no adverse effects following 45 days of oral administration, based upon criteria of body weight, appearance and behavior (5). Microarray analysis of GO-Y030 and curcumin-treated cells produced similar expression profiles, with the expression of the majority of affected genes falling within a 2-fold variation. At the protein level however, GO-Y030 was found to down-regulate known contributors to oncogenesis such as B-catenin, HER2/neu (also known as ErbB-2), c-Myc and cyclin D1 at dosages far lower than those required to elicit a comparable effect with curcumin (5). Given the multitude of known molecular targets of curcumin, it seems likely that GO-Y030 may have a similar effect on other oncoproteins that have yet to be described. 
A potential target is the signal transducer and activator of tanscription 3 (STAT3), a transcription factor whose activity can also be down-regulated by curcumin $(6,7)$. Persistently active STAT3 is found with high frequency in a wide range of human cancer cell lines and tissues where it has been implicated in stimulating cell proliferation, promoting angiogenesis, mediating immune evasion, and conferring increased resistance to apoptosis (8-13). These activities are apparently required for the continued survival of certain cancer cells, as interference with the STAT3 pathway results in growth inhibition and the induction of apoptosis (14-16). Although knock-out of STAT3 is lethal to mice in early embryogenesis, it appears to be dispensable in most normal adult cell and tissue types $(17,18)$. As such, STAT3 has emerged as an attractive chemotherapeutic target $(19,20)$.

In our present study, we compared the effects of GO-Y030 and curcumin in breast and pancreatic cancers that feature constitutively activated STAT3. As hypothesized, GO-Y030 was substantially more effective than curcumin at inhibiting the viability of these cells and inducing apoptosis in vitro. It was also revealed that GO-Y030 was more capable of preventing the phosphorylation and subsequent activation of STAT3, an observation that may partially explain why GOY030 is more effective than curcumin against certain cancer cells.

\section{Materials and methods}

Cell culture. The human breast cancer cell line MDA-MB-231 and three human pancreatic cancer cell lines (PANC-1, BXPC-3, and HPAC) were acquired from the American Type Culture Collection. These cells were maintained in 1X Dulbecco's modification of Eagle's medium (DMEM) supplemented with $10 \%$ fetal bovine serum (FBS) (Invitrogen), $4.5 \mathrm{~g} / \mathrm{l}, \mathrm{L}$-glutamine, and sodium pyruvate (Mediatech) and $1 \%$ penicillin/streptomycin $(\mathrm{P} / \mathrm{S})$. The cells were maintained in incubators set at $37^{\circ} \mathrm{C}$ and aired with $5 \% \mathrm{CO}_{2}$.

Western blot analysis. GO-Y030 and curcumin (SigmaAldrich, St. Louis, MO) were dissolved in sterile dimethylsulfoxide (DMSO) to make $20 \mathrm{mM}$ stock solutions. The MDA-MB-231 breast cancer cells and PANC-1, BXPC-3, and HPAC pancreatic cancer cells were grown to semiconfluency and treated with $1.0,2.5$, and $5 \mu \mathrm{M}$ of GO-Y030, or $2.5-20 \mu \mathrm{M}$ of curcumin for $24 \mathrm{~h}$. For Western blots, $50 \mu \mathrm{g}$ of total cell lysates were resolved by SDS polyacrylamide gel electrophoresis (PAGE) and transferred to PVDF membrane. These membranes were then blotted with phospho-specific STAT3 antibody (Tyrosine 705; \#9131 Cell Signaling Technology, Beverly, MA), phospho-independent STAT3 antibody (\#9132 Cell Signaling Tech.), cleaved PARP antibody (\#9546 Cell Signaling Tech.), cleaved caspase-3 (Asp175) antibody (\#9661 Cell Signaling Tech.), and GAPDH antibody (MAB374 Chemicon International Inc., Temecula, CA). Membranes were analyzed with enhanced chemiluminescence Plus reagents (GE Healthcare UK Ltd., Buckinghamshire, UK) and scanned with a Storm phosphorimager (Amersham Pharmacia Biotech Inc., Piscataway, NJ).

MTT cell viability assay. MDA-MB-231, PANC-1, BXPC-3, and HPAC breast and pancreatic cancer cells were seeded in 96-well plates (4000 cells/well) in triplicate in $10 \%$ FBS DMEM. After $24 \mathrm{~h}$, the cells were treated with $0.5-5 \mu \mathrm{M}$ of GO-Y030 or $0.5-30 \mu \mathrm{M}$ of curcumin for $72 \mathrm{~h}$. MTT (NPI Research Products International Corp., Mt. Prospect, IL) diluted in sterile PBS was then added to each well to a final concentration of $5 \mathrm{mg}$ MTT per ml. The cells were incubated for $3.5 \mathrm{~h}$ at $37^{\circ} \mathrm{C}$. Reactions were stopped by the addition of $100 \mu \mathrm{l}$ of solubilization solution (a 1:1 mixture of $\mathrm{ddH}_{2} \mathrm{O}$ and $\mathrm{N}, \mathrm{N}$-dimethylformamide with $20 \%$ SDS, $\mathrm{pH} 4.5$ ). The plates were kept in the dark at room temperature overnight before being read at $570 \mathrm{~nm}$ in a spectrophotometer. Half-maximal inhibitory concentrations $\left(\mathrm{IC}_{50}\right)$ were determined using Sigma Plot 9.0 software (Systat Software Inc., San Jose, CA) using the 4 parameter logistic function standard curve analysis for dose response.

STAT3-dependent transcriptional luciferase assay. MDAMB-231 breast cancer cells were stably transfected with the pLucTKS3 luciferase reporter, a construct that contains seven copies of the STAT3 binding site in a thymidine kinase minimal promoter. Expression of luciferase is contingent upon activation of STAT3 (21). These cells were grown to semiconfluency in 6-well plates and then treated with the listed concentrations of curcumin or GO-Y030 for $24 \mathrm{~h}$ in 5\% FBS DMEM. The cells were washed with PBS and harvested in reporter lysis buffer. Lysate $(20 \mu \mathrm{l})$ was used in the luciferase per the manufacturer's protocol (Promega, Madison, WI). Samples were run in triplicate. The data were normalized via a BCA protein assay (Thermo Scientific, Rockford, IL) to determine the total amount of protein present in each lysate. Luciferase activity is expressed as CPS per $\mu \mathrm{g}$ protein. Curcumin- and GO-Y030-treated STAT3 luciferase activity is presented relative to a pLucTKS3-transfected but otherwise untreated sample, arbitrarily set at $100 \%$. Error bars represent \pm standard deviation.

Soft agar colony formation assay. A $0.6 \%$ agar gel with $10 \%$ FBS in DMEM was prepared and added to the wells of a 6-well culture dish as a base agar. MDA-MB-231 breast cancer cells (5000 per well) were plated for anchorage-independent growth analysis in $0.4 \%$ agar gel with $10 \%$ FBS in DMEM supplemented with the target treatment on-top of the base agar. The cells were allowed to grow at $37^{\circ} \mathrm{C}$ for 2 weeks. The effect of the drugs on anchorage independent growth was determined by colony growth. Colonies were stained with MTT dye (100 $\mu 1$ per well) and pictures were taken with a Leica MZ 16FA inverted microscope (Leica Microsystems, Wetzlar, Germany) with a 7.4 Slider Camera (Diagnostic Instruments Inc., Sterling Heights, MI). Colonies were scored by counting with an inverted microscope. Colony formation in each treatment is expressed as a percentage of the total colonies formed in the untreated control.

\section{Results}

GO-Y030 is a more potent inhibitor of viability in human breast and pancreatic cancer cells. In this study, we initially examined the growth suppressive activities of GO-Y030 and curcumin (Fig. 1) in human breast and pancreatic cancer cell lines. MDA-MB-231 is basal-like triple-negative (in regards 
<smiles>COc1cc(/C=C/C(=O)CC(=O)/C=C/c2ccc(O)c(OC)c2)ccc1O</smiles><smiles>COCOc1cc(/C=C/C(=O)/C=C/c2cc(OCOC)cc(OCOC)c2)cc(OCOC)c1</smiles>

Figure 1. The chemical structures of curcumin and GO-Y030.

Table I. $\mathrm{IC}_{50}$ values for cell viability in human breast and pancreatic cancer cells.

MDA-MB-231 HPAC PANC-1 BXPC-3

\begin{tabular}{lrrrr}
\hline Curcumin & 19.3 & 16.7 & 27.8 & 8.7 \\
GO-Y030 & 1.2 & 2.2 & 0.1 & 0.2 \\
\hline
\end{tabular}

${ }^{a}$ Cancer cells were treated for $72 \mathrm{~h}$ and cell viability was analyzed by MTT assays. $\mathrm{IC}_{50}$ values $(\mu \mathrm{M})$ were subsequently determined for each cell line.

to presence of the estrogen, progesterone, and human epidermal growth factor type 2 (HER2) receptors) breast carcinoma cell line that features high endogenous levels of phosphorylated STAT3 (pSTAT3) (22). We assessed the viability of these cells with MTT assays following $72 \mathrm{~h}$ of treatment with different concentrations of either curcumin or GO-Y030. Although viability of the MDA-MB-231 breast cancer cells was reduced in a dose-dependent fashion following treatment with each compound, the inhibitory effects of GO-Y030 were much more pronounced. The computation of $\mathrm{IC}_{50}$ values from these data suggests that GO-Y030 is several times more effective than curcumin (1.2 versus $19.3 \mu \mathrm{M}$ respectively) in regards to its impact on general viability (Table I).
We also examined GO-Y030- and curcumin-induced inhibition of viability in three human pancreatic cancer cell lines: PANC-1, BXPC-3, and HPAC. PANC-1 is a pancreatic epitheloid carcinoma with high levels of endogenous pSTAT3, whereas both BXPC-3 and HPAC are adenocarcinoma cell lines with elevated levels of pSTAT3 $(23,24)$. In accordance with our observations in MDA-MB-231, GO-Y030 was again the more potent of the two compounds in each of the pancreatic cell lines evaluated (Table I).

GO-Y030 induces apoptosis and inhibits STAT3 phosphorylation more effectively than curcumin in breast and pancreatic cancer cells. We then examined if GO-Y030 and curcumin were leading to the induction of apoptosis by assaying for the presence of cleaved poly-(ADP-ribose) polymerase (PARP) in cancer cells following their exposure to these compounds. PARP is an early target of active caspases, and its cleavage product can serve as a marker of apoptosis (25). Based on the higher efficacy of GO-Y030 observed in the viability assays, we decided to treat MDA-MB-231 cells with 1-5 $\mu \mathrm{M}$ concentrations of GO-Y030 and 5-20 $\mu \mathrm{M}$ concentrations of curcumin for $24 \mathrm{~h}$. Western blot analysis with a cleavage-specific PARP antibody revealed that induction of apoptosis can be detected in MDA-MB-231 cells at GO-Y030 concentrations as low as 2.5 and $5 \mu \mathrm{M}$ (Fig. 2A). Cleavage of PARP was also detected in HPAC and PANC-1 pancreatic cancer lines at these concentrations of GO-Y030, whereas curcumin concentrations as high as $20 \mu \mathrm{M}$ failed to induce
A.

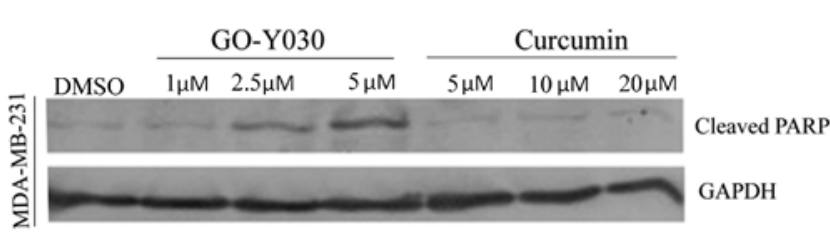

B.

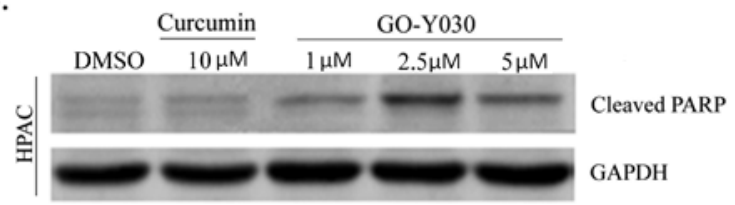

C.

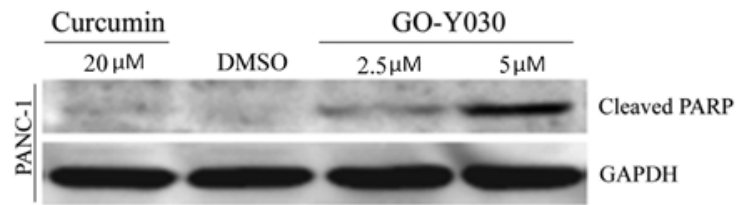

D.

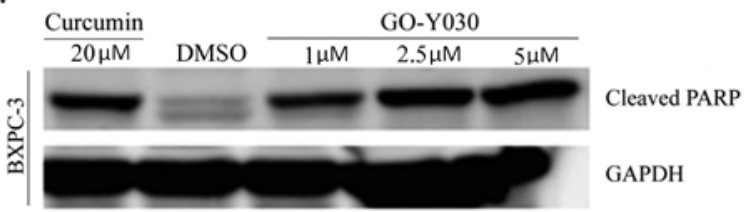

Figure 2. GO-Y030 induces cleavage of PARP in (A) the MDA-MB-231 breast cancer cell line, and the pancreatic cancer cell lines (B) HPAC, (C) PANC-1, and (D) BXPC-3. Total cell lysate $(50 \mu \mathrm{g})$ from each sample was resolved by SDS-PAGE and transferred to membranes for Western bblotting with antibodies for cleaved PARP or GAPDH as a loading control. 


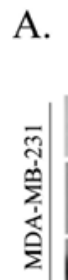
DMSO $\frac{\text { GO-Y030 }}{1 \mu \mathrm{M} \quad 2.5 \mu \mathrm{M} \quad 5 \mu \mathrm{M}} \frac{\text { Curcumin }}{5 \mu \mathrm{M} \quad 10 \mu \mathrm{M} \quad 20 \mu \mathrm{M}}$

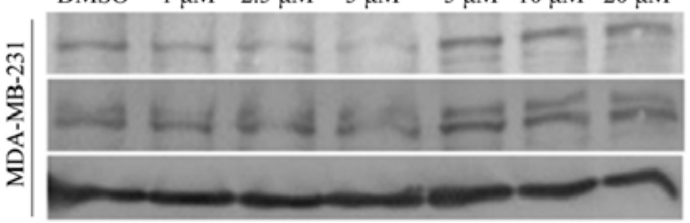

pSTAT3 (Y705)

STAT3

GAPDH
C.

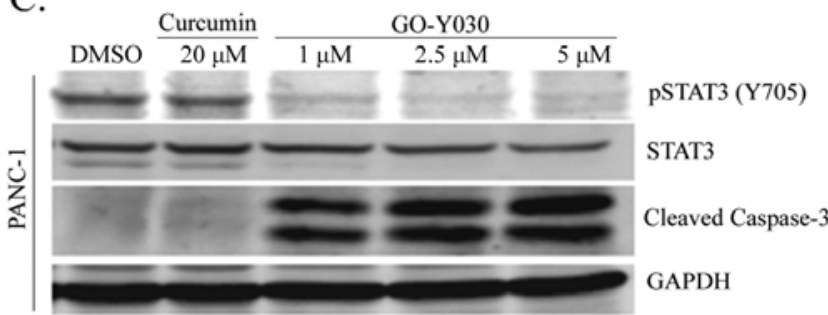

B.

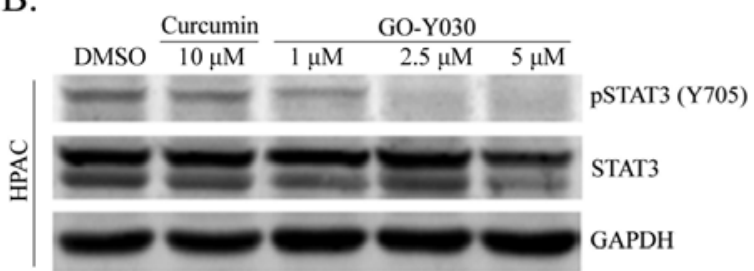

D.

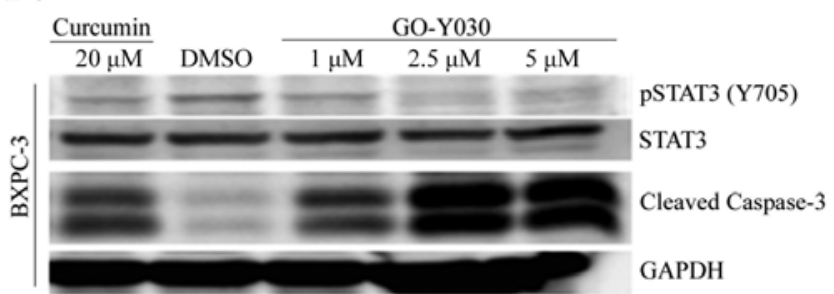

Figure 3. GO-Y030 is a more potent inhibitor of STAT3 phosphorylation than curcumin. Semi-confluent plates of MDA-MB-231, HPAC, PANC-1 and BXPC-3 cells were treated with the listed concentrations of these compounds for $24 \mathrm{~h}$. Total cell lysate $(50 \mu \mathrm{g})$ from each sample was resolved by SDS-PAGE and transferred to membranes for Western blotting with antibodies specific for pSTAT3, phospho-independent STAT3, cleaved caspase-3 (Asp175), or GAPDH.

A. Luciferase activity in MDA-MB-231

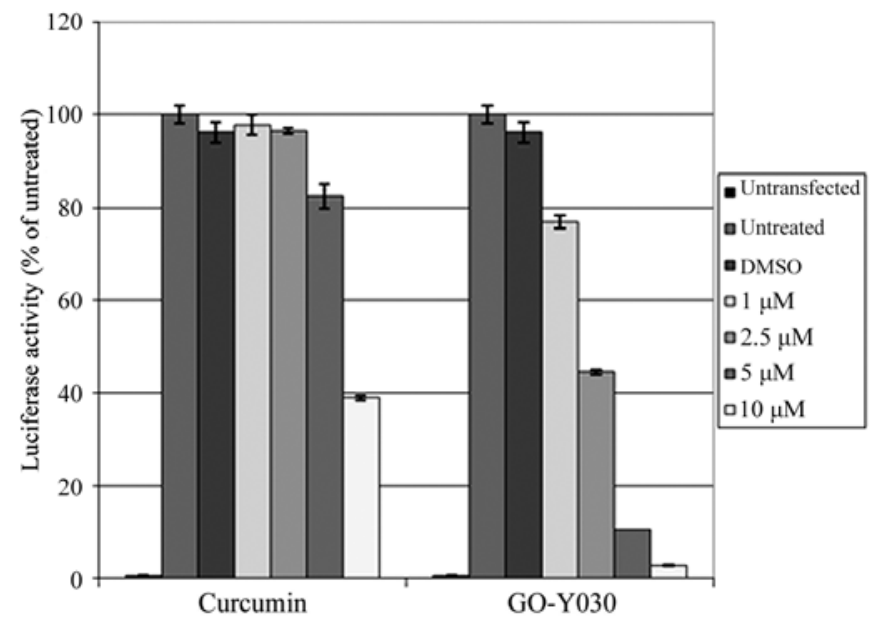

B. Colony formation in MDA-MB-231

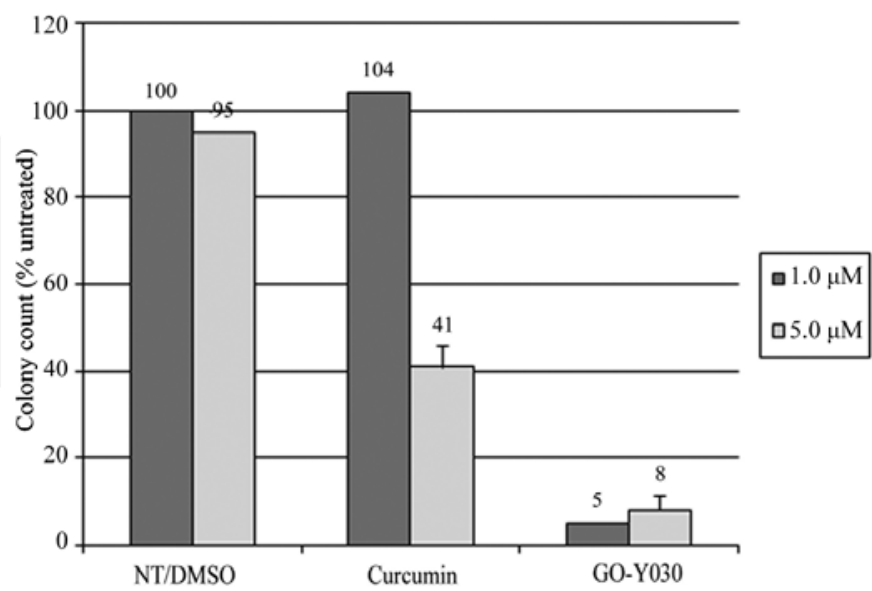

Figure 4. (A) GO-Y030 and curcumin on interfere with STAT3-mediated transcription of luciferase in a dose-dependent manner. MDA-MB-231 breast cancer cells were stably transfected with the pLucTKS3 luciferase construct, whose expression of luciferase is contingent upon activated pSTAT3. These cells were seeded and treated with the listed concentrations of curcumin or GO-Y030 in triplicate for $24 \mathrm{~h}$. Relative luciferase activity was measured and normalized as described in Materials and methods. (B) The ability of MDA-MB-231 breast cancer cells to form colonies in soft agar is decreased following treatment with $5 \mu \mathrm{M}$ of curcumin or 1 and $5 \mu \mathrm{M}$ of GO-Y030. The colony numbers were counted following 2 weeks of growth time.

cleavage of PARP (Fig. 2B and C). Treatment of the BXPC-3 pancreatic cancer line with $1-5 \mu \mathrm{M}$ concentrations of GO-Y030 produced nearly identical results, however this cell line also appeared to be more sensitive to high dosages of curcumin (Fig. 2D).

To determine if GO-Y030 was having any impact on the phosphorylation status of STAT3, we performed a similar series of experiments and assayed for the presence of pSTAT3 using a phospho-specific antibody. MDA-MB-231 breast cancer cells were treated with GO-Y030 or curcumin for
$24 \mathrm{~h}$. A $5 \mu \mathrm{M}$ concentration of GO-Y030 was sufficient to inhibit STAT3 phosphorylation whereas concentrations as high as $20 \mu \mathrm{M}$ of curcumin had little to no effect (Fig. 3A). Phosphorylation of STAT3 was also examined in the HPAC, PANC-1, and BXPC-3 pancreatic cell lines. GO-Y030 noticeably reduced levels of pSTAT3 at concentrations as low as $1 \mu \mathrm{M}$, and the presence of pSTAT3 was nearly undetectable in samples treated with concentrations of 2.5 and $5 \mu \mathrm{M}$ across all three pancreatic cancer cell lines tested (Fig. 3B and D). Treatment with equivalent dosages of curcumin 
elicited no observable effect on levels of pSTAT3 in these cells (data not shown). Some reduction in pSTAT3 was seen following treatment with 10 and $20 \mu \mathrm{M}$ concentrations of curcumin, the extent of which varied with the cell line.

GO-YO3O inhibits STAT3-mediated transcription of luciferase. A luciferase assay was employed to gauge GO-Y030-mediated repression of STAT3 transcriptional activity. Due to its high endogenous levels of pSTAT3, MDA-MB-231 breast cancer cells were chosen to be stably transfected with pLucTKS3, a luciferase construct that features seven copies of the STAT3 binding site in a thymidine kinase minimal promoter. Expression of luciferase is thus contingent upon the phosphorylation and activation of STAT3 (22). These stably transfected cells were treated with 1-10 $\mu \mathrm{M}$ of GO-Y030 or curcumin for $24 \mathrm{~h}$. Luciferase activity was monitored via a luminometer, and the luminescence of the GO-Y030- and curcumin-treated cells was compared to that of an untreated control. Following normalization of the data, both compounds were shown to reduce the expression of luciferase in a dose-dependent manner, however GO-Y030 was again much more effective in this capacity (Fig. 4A). These results confirm that GO-Y030 is a potent agent of inhibiting STAT3 phosphorylation and STAT3-dependent transcriptional luciferase activity.

GO-Y030 is a potent inhibitor of anchorage-independent growth. The ability of transformed cells to grow and proliferate in the absence of substratum attachment is one of the hallmarks of malignancy and is vitally important in the formation of the tumor (26). Since curcumin has been shown to down-regulate STAT3 phosphorylation (7), which may have an effect on anchorage independency (27), we hypothesized that curcumin would lead to a decrease in the number of colonies formed in soft agar and that GO-Y030 would show an even greater decrease. Our results show that both curcumin and GO-Y030 treatment led to decreased colony formation in soft agar when compared to the untreated control in MDA-MB-231 breast cancer cells. Compared to the control, samples treated with a $5 \mu \mathrm{M}$ concentration of curcumin showed a nearly $60 \%$ decrease in the number of colonies formed. Concentrations of 1 and $5 \mu \mathrm{M}$ GO-Y030 showed a 95 and $92 \%$ decrease in colony number respectively (Fig. 4B). These data are indicative that in addition to reducing overall viability, curcumin and to a larger extent GO-Y030 inhibit the ability of cancer cells to grow in an anchorage-independent manner.

\section{Discussion}

The American Cancer Society estimates that 180,000 new cases of invasive breast cancer are diagnosed each year. It remains the second most fatal form of cancer in women after those that afflict the lung and bronchus, and it is responsible for approximately 40,000 deaths annually (28). Although cancers of the exocrine pancreas are comparatively less common, they remain among the most deadly; the overall survival rate of pancreatic cancer patients is $<4 \%$, and an estimated 33,370 deaths resulted from the disease in the year 2007 alone (28). As such, there is a critical need for better approaches to the treatment of these types of cancers.

Numerous reports have shown that curcumin can inhibit multiple oncogenic processes in many cancers, including those of the breast and pancreas. The low bioavailability of curcumin limits its use in therapeutic applications, however, necessitating alternative routes of administration. In this study, we further evaluated the inhibitory effects of the curcumin analogue GO-Y030. Our data clearly demonstrate the increased efficacy of this compound over curcumin in inhibiting breast and pancreatic cancer cell viability. Moreover, GO-Y030 was found to induce apoptosis in these cells at concentrations far lower than those required to elicit a comparable effect following curcumin treatment.

We also show that GO-Y030 is a potent inhibitor of STAT3. Constitutively activated STAT3 has been reported in a high percentage of cancers, where it contributes to increased cell growth, apoptotic resistance, angiogenesis and enhanced metastatic potential (8). Treatment of breast and pancreatic cancer cells with GO-Y030 resulted in moderate to sharp declines of detectable levels of pSTAT3, showing some variance across cell lines. Luciferase assay analysis with an activated STAT3-dependent luciferase construct revealed that GO-Y030 could effectively suppress STAT3 transcription in a dose-dependent manner, and low dosages of GO-Y030 were found to impair the ability of MDA-MB-231 cells to form colonies in soft agar. Although further analysis of the GO-Y030 inhibitory effects is warranted, our results thus far show that GO-Y030 is a potent agent to suppress viability and anchorage independency, induce apoptosis, and inhibit constitutive STAT3 signaling. It may thus have translational potential as a chemopreventative or therapeutic agent for human cancer, pending further examination of its bio-availability and tissue distribution.

\section{Acknowledgements}

This work was supported in part by a Susan Komen Breast Cancer Research grant, a National Foundation for Cancer Research grant to Jiayuh Lin and a NIH R03 grant CA13747901A1.

\section{References}

1. Goel A, Kunnumakkara AB and Aggarwal BB: Curcumin as 'Curecumin': from kitchen to clinic. Biochem Pharmacol 75: 787-809, 2008.

2. Hatcher H, Planalp R, Cho J, Torti FM and Torti SV: Curcumin: from ancient medicine to current clinical trials. Cell Mol Life Sci (In press).

3. Anand P, Kunnumakkara AB, Newman RA and Aggarwal BB: Bioavailability of curcumin: problems and promises. Mol Pharm 4: 807-818, 2007.

4. Mosley CA, Liotta DC and Snyder JP: Highly active anticancer curcumin analogues. Adv Exp Med Biol 595: 77-103, 2007.

5. Ohori H, Yamakoshi H, Tomizawa M, et al: Synthesis and biological analysis of new curcumin analogues bearing an enhanced potential for the medicinal treatment of cancer. Mol Cancer Ther 5: 2563-2571, 2006.

6. Aggarwal BB and Shishodia S: Molecular targets of dietary agents for prevention and therapy of cancer. Biochem Pharmacol 71: 1397-1421, 2006

7. Bharti A, Donato N and Aggarwal B: Curcumin (diferuloylmethane) inhibits constitutive and IL-6-inducible STAT3 phosphorylation in human multiple myeloma cells. J Immunol 171: 3863-3871, 2003.

8. Yu H and Jove R: The STATs of cancer - new molecular targets come of age. Nat Rev Cancer 4: 97-105, 2004

9. Alas S and Bonavida B: Inhibition of constitutive STAT3 activity sensitizes resistant non-Hodgkin's lymphoma and multiple myeloma to chemotherapeutic drug-mediated apoptosis. Clin Cancer Res 9: 316-326, 2003. 
10. Buettner R, Mora L and Jove R: Activated STAT signaling in human tumors provides novel molecular targets for therapeutic intervention. Clin Cancer Res 8: 945-954, 2002.

11. Shen Y, Devgan G, Darnell JJ and Bromberg J: Constitutively activated Stat 3 protects fibroblasts from serum withdrawal and UV-induced apoptosis and antagonizes the proapoptotic effects of activated Stat1. Proc Natl Acad Sci USA 98: 1543-1548, 2001.

12. Real P, Sierra A, De Juan A, Segovia J, Lopez-Vega J and Fernandez-Luna J: Resistance to chemotherapy via Stat3dependent overexpression of $\mathrm{Bcl}-2$ in metastatic breast cancer cells. Oncogene 21: 7611-7618, 2002.

13. Wang T, Niu G, Kortylewski M, et al: Regulation of the innate and adaptive immune responses by Stat-3 signaling in tumor cells. Nat Med: 48-54, 2004.

14. Aoki Y, Feldman G and Tosato G: Inhibition of STAT3 signaling induces apoptosis and decreases survivin expression in primary effusion lymphoma. Blood 101: 1535-1542, 2003

15. Burke W, Jin X, Liu R, Huang M, Reynolds RK and Lin J: Inhibition of constitutively active Stat 3 pathway in ovarian and breast cancer cells. Oncogene 20: 7925-7934, 2001

16. Calvin D, Nam S, Buettner R, Sekharam M, Torres-Roca J and Jove R: Inhibition of STAT3 activity with STAT3 antisense oligonucleotide (STAT3-ASO) enhances radiation-induced apoptosis in DU145 prostate cancer cells. Int J Radiat Oncol Biol Phys 57: S297, 2003.

17. Takeda K, Noguchi K, Shi W, et al: Targeted disruption of the mouse Stat 3 gene leads to early embryonic lethality. Proc Natl Acad Sci USA 94: 3801-3804, 1997.

18. Akira S: Roles of STAT3 defined by tissue-specific gene targeting. Oncogene 19: 2607-2611, 2000.

19. Germain D and Frank DA: Targeting the cytoplasmic and nuclear functions of signal transducers and activators of transcription 3 for cancer therapy. Clin Cancer Res 13: 5665-5669, 2007.
20. Aggarwal BB, Sethi G, Ahn KS, et al: Targeting signaltransducer-and-activator-of-transcription-3 for prevention and therapy of cancer: modern target but ancient solution. Ann NY Acad Sci 1091: 151-169, 2006.

21. Turkson J, Bowman T, Garcia R, Caldenhoven E, De Groot RP and Jove R: Stat 3 activation by src induces specific gene regulation and is required for cell transformation. Mol Cell Biol 18: 2545-2552, 1998.

22. Song H, Wang R, Wang S and Lin J: A low-molecular-weight compound discovered through virtual database screening inhibits Stat3 function in breast cancer cells. Proc Natl Acad Sci USA 102: 4700-4705, 2005.

23. Wei D, Le X, Zheng L, et al: Stat3 activation regulates the expression of vascular endothelial growth factor and human pancreatic cancer angiogenesis and metastasis. Oncogene 22: 319-329, 2003.

24. Kotha A, Sekharam M, Cilenti L, et al: Resveratrol inhibits Src and Stat 3 signaling and induces the apoptosis of malignant cells containing activated Stat3 protein. Mol Cancer Ther 5: 621-629, 2006.

25. Scovassi AI and Poirier GG: Poly(ADP-ribosylation) and apoptosis. Mol Cell Biochem 199: 125-137, 1999.

26. Hanahan D and Weinberg RA: The hallmarks of cancer. Cell 100: 57-70, 2000 .

27. Schlessinger K and Levy DE: Malignant transformation but not normal cell growth depends on signal transducer and activator of transcription 3. Cancer Res 65: 5828-5834, 2005.

28. Jemal A, Siegel R, Ward E, et al: Cancer statistics, 2008. CA Cancer J Clin 58: 71-96, 2008. 